TITLE:

\title{
Effects of loading frequency on the functional adaptation of trabeculae predicted by bone remodeling simulation.
}

$\operatorname{AUTHOR}(S)$ :

Kameo, Yoshitaka; Adachi, Taiji; Hojo, Masaki

\section{CITATION:}

Kameo, Yoshitaka ... [et al]. Effects of loading frequency on the functional adaptation of trabeculae predicted by bone remodeling simulation.. Journal of the mechanical behavior of biomedical materials 2011, 4(6): 900-908

\section{ISSUE DATE:}

2011-08

URL:

http://hdl.handle.net/2433/152436

\section{RIGHT:}

(C) 2011 Elsevier Ltd.; This is not the published version. Please cite only the published version.; この論文は出版社版でありません。引用の際に は出版社版をご確認ご利用ください。 


\section{Effects of loading frequency on the functional adaptation of trabeculae predicted by bone remodeling simulation}

Yoshitaka Kameo $^{\text {a, b }}$, Taiji Adachi ${ }^{\text {b, c }}$, and Masaki Hojo ${ }^{\text {a }}$

a: Department of Mechanical Engineering and Science, Kyoto University

b: Computational Cell Biomechanics Team, VCAD System Research Program, RIKEN

c: Department of Biomechanics, Institute for Frontier Medical Sciences, Kyoto University

Corresponding author: Taiji Adachi, Ph.D.

Mailing Address: Department of Biomechanics,

Institute for Frontier Medical Sciences,

Kyoto University

53, Syogoin-kawaramachi, Sakyo, Kyoto 606-8507, Japan

Telephone \& Fax: $\quad$ +81 (75) 751-4853

E-mail: $\quad$ adachi@frontier.kyoto-u.ac.jp

Submitted to Journal of the Mechanical Behavior of Biomedical Materials

Key words: Bone remodeling, Functional adaptation, Loading frequency, Cellular

mechanosensing, Computational simulation 


\section{Abstract}

The process of bone remodeling is regulated by metabolic activities of many bone cells.

While osteoclasts and osteoblasts are responsible for bone resorption and formation,

respectively, activities of these cells are believed to be controlled by a mechanosensory

system of osteocytes embedded in the extracellular bone matrix. Several experimental and theoretical studies have suggested that the strain-derived interstitial fluid flow in lacuno-canalicular porosity serves as the prime mover for bone remodeling. Previously, we constructed a mathematical model for trabecular bone remodeling that interconnects the microscopic cellular activities with the macroscopic morphological changes in trabeculae through the mechanical hierarchy. This model assumes that fluid-induced shear stress acting on osteocyte processes is a driving force for bone remodeling. The validity of this model has been demonstrated with a remodeling simulation using a two-dimensional trabecular model. In this study, to investigate the effects of loading frequency, which is thought to be a significant mechanical factor in bone remodeling, we simulated morphological changes of a three-dimensional single trabecula under cyclic uniaxial loading with various frequencies. The results of the simulation show the trabecula reoriented to the loading direction with the progress of bone remodeling. Furthermore, as the imposed loading frequency increased, the diameter of the trabecula in the equilibrium state was enlarged by remodeling. These results indicate that our simulation model can successfully evaluate the relationship between loading frequency and trabecular bone remodeling. 


\section{Introduction}

Bone is a load-bearing tissue that can adapt its internal structure and outer shape by remodeling to a changing mechanical environment. The morphological changes of the trabecular microstructure are realized by the coupling of osteoclastic bone resorption and osteoblastic bone formation (Parfitt, 1994). It is widely believed that the metabolic activities of these executive cells are coupled with osteocytes, which are interconnected within the extracellular bone matrix, forming a three-dimensional intercellular network through cellular processes (Cowin et al., 1991; Donahue et al., 1995; Kamioka et al., 2001, 2009; Knothe Tate et al., 2004; Sugawara et al., 2005). The osteocyte network is considered to have an important role in mechanosensing and intercellular signal transmission (Mullender and Huiskes, 1997; Burger and Klein-Nulend, 1999; Cowin, 2007; Huo et al, 2008; Adachi et al., 2009a, b, c).

Although the mechanism of cellular mechanosensing is not well understood, attempts have been made to determine the mechanical stimulus that derives the responses of mechanosensor cells. A variety of mechanical stimuli, such as strain energy density (Mullender et al., 1994; Huiskes et al., 2000; Mullender and Huiskes, 1995; Ruimerman at al., 2005), micro-damage (Prendergast and Taylor, 1994; McNamara and Prendergast, 2007; Mulvihill and Prendergast, 2008), stress nonuniformity (Adachi et al., 1997, 2001; Tsubota and Adachi, 2004, 2005, 2006; Tsubota et al., 2002, 2009), and fluid flow (Weinbaum et al., 1994; Burger and Klein-Nulend, 1999; Knothe Tate et al., 1998; Klein-Nulend et al., 2005; Bonewald and Johnson, 2008; Fritton and Weinbaum, 2009), are assumed to be candidates for a remodeling stimulus. Experimental studies indicate that a significant mechanical factor 
affecting bone remodeling is the strain rate rather than the strain itself (Fritton et al., 2000; Goldstein et al., 1991; Lanyon, 1984). The theoretical study based on poroelasticity (Weinbaum et al., 1994) suggests that the interstitial fluid flow in lacuno-canalicular porosity induced by strain plays an important role in osteocyte mechanosensing. In the microstructure model of Weinbaum et al. (1994), fluid-induced shear stress over the osteocytic process is considered to be a driving force for bone remodeling. This model was later refined, indicating the importance of cellular attachment to canalicular projections (You et al., 2001; Weinbaum et al., 2003; Han et al., 2004) and the fiber connection between the cell membrane and the canalicular wall (Wang et al., 2007) as well as the fluid flow. Thus, the progress in this field of research from the macroscopic to microscopic level has provided important information about the mechanism of cellular mechanosensing.

A number of computational models for bone remodeling using finite element analysis have been proposed to investigate the mechanism of bone self-regulation (Cowin, 1993; Huiskes et al., 1987, 2000; Jang and Kim, 2008; Coelho et al., 2009; Dunlop et al., 2009; Martinez-Reina et al., 2009; Gerhard et al., 2009). We have developed a mathematical model for trabecular surface remodeling by assuming the nonuniformity of local stress on the trabecular surface as a driving force for remodeling (Adachi et al., 1997, 2001; Tsubota and Adachi, 2004, 2005, 2006; Tsubota et al., 2002). Applying this phenomenological model to the trabecular remodeling simulation for an entire human proximal femur, we were able to successfully describe a three-dimensional trabecular pattern similar to that in the actual femur (Tsubota et al., 2009). However, this model does not explicitly consider the possible 
mechanisms at the cellular level, even though bone remodeling is accomplished by the coupling of the cellular activities of osteoclasts, osteoblasts, and osteocytes.

In order to explain the macroscopic tissue level changes in trabecular morphology in terms of the microscopic cellular level activities, we recently proposed a mathematical model for bone remodeling, taking cellular mechanosensing and intercellular communication into consideration (Adachi et al., 2010). This model is based on the hypothesis that osteocytes respond to fluid-induced shear stress (Weinbaum et al., 1994; Klein-Nulend et al., 1995a, b) and deliver their mechanical signals to the surface cells by intercellular communication (Adachi et al., 2009c). As a result, bone formation and resorption are regulated. The validity of our model was demonstrated by a remodeling simulation for a two-dimensional trabecular model (Adachi et al., 2010). The purpose of this study is to investigate the effects of loading frequency, which is thought to be a significant mechanical factor for bone remodeling. By applying the mathematical model for bone remodeling (Adachi et al., 2010) to the three-dimensional voxel finite element model of a trabecula, we performed bone remodeling simulation for a single trabecula subjected to cyclic uniaxial loading with various frequencies.

\section{Methods}

\subsection{Mathematical model of trabecular bone remodeling}

\section{Theoretical framework}

The proposed theoretical framework for trabecular bone remodeling is illustrated in Fig. 1. Mechanical loading applied to bone tissue induces bone matrix deformation and interstitial 
fluid flow. The mechanical behavior of the interstitial fluid can be quantified by the fluid pressure $p$ and its gradient $4 p$. The osteocytes buried in the bone matrix or the cells on the trabecular surface, such as osteoblasts and bone lining cells, respond to the fluid flow and transduce the mechanical stimulus into the biochemical signal $S_{\mathrm{oc}}$. Then, the produced signal is transmitted to the trabecular surface through an intercellular network system and is integrated as the total stimulus $S_{\text {sf. }}$ Finally, the rate of trabecular surface remodeling $\dot{M}$, which expresses formation and resorption of the trabecular surface, is regulated by the stimulus $S_{\text {sf }}$.

While the process of generating interstitial fluid flow by applied loading is purely mechanical, the other three processes, i.e., cellular mechanosensing, intercellular communication, and trabecular surface remodeling, are achieved by the intricate mechanical-biochemical coupling phenomena, and their detailed mechanisms remain unproven. In the following subsections, the mathematical models of each process are constructed based on rational assumptions.

\section{Cellular mechanosensing}

It is believed by many researchers that osteocytes in the bone matrix have a role as mechanosensory cells, and shear stress caused by interstitial fluid flow over the osteocytes processes can be considered to be a mechanical stimulus. The evaluation of the shear stress was based on the microstructure model of Weinbaum et al. (1994) shown in Fig. 2. This model accounts for the interstitial fluid flow through a fiber matrix, such as proteoglycan, in 
an annular canaliculus. Assuming the fluid flow is governed by the Brinkman equation, the shear stress $\tau_{\mathrm{p}}(\boldsymbol{n})$, acting on the osteocyte processes that align in direction $\boldsymbol{n}$, can be derived using the fluid pressure gradient at bone tissue level $\nabla p$ as:

$\tau_{\mathrm{p}}(\boldsymbol{n})=\frac{q r_{\mathrm{p}}}{\gamma}\left[A_{1} I_{1}\left(\frac{\gamma}{q}\right)-B_{1} K_{1}\left(\frac{\gamma}{q}\right)\right] \nabla p(\boldsymbol{x}) \cdot \boldsymbol{n}$,

where constants $A_{1}$ and $B_{1}$ are given by

$$
\begin{aligned}
& A_{1}=\frac{K_{0}(\gamma)-K_{0}(\gamma / q)}{I_{0}(\gamma / q) K_{0}(\gamma)-I_{0}(\gamma) K_{0}(\gamma / q)} \\
& B_{1}=\frac{I_{0}(\gamma / q)-I_{0}(\gamma)}{I_{0}(\gamma / q) K_{0}(\gamma)-I_{0}(\gamma) K_{0}(\gamma / q)}
\end{aligned}
$$

In these equations, $q$ is the ratio of the radius of the canaliculus $r_{\mathrm{c}}$ to that of the process $r_{\mathrm{p}}$ $\left(q=r_{\mathrm{c}} / r_{\mathrm{p}}\right), \gamma$ is the dimensionless parameter defined by $\gamma=r_{\mathrm{c}} / \sqrt{k_{\mathrm{p}}}$, and $I_{0}, I_{1}, K_{0}$, and $K_{1}$ are modified Bessel functions (Weinbaum et al., 1994). The constant $k_{\mathrm{p}}$ is the permeability of the fiber matrix in the fluid annulus and can be approximated by the equation $0.0572 a_{0}^{2}\left(\Delta / a_{0}\right)^{2.377}$ using the fiber radius $a_{0}$ and the spacing of the fiber matrix $\Delta$ (Tsay and Weinbaum, 1991). The fluid pressure gradient $\nabla p$ is calculated using poroelastic finite element analysis (Detournay and Cheng, 1993; Manfredini et al., 1999).

In this study, it was assumed that osteocytes are susceptible to the average value of fluid-induced shear stress in a day, and the quantity of biochemical signals that osteocytes produce is equivalent to the value of the integral of the average shear stress on the cell processes. Introducing the volume fraction of canaliculi oriented to $\boldsymbol{n}, \rho_{\mathrm{c}}(\boldsymbol{n})$, at a particular position $\boldsymbol{x}$ (Kameo et al., 2010), the signal $S_{\mathrm{oc}}(\boldsymbol{x})$ produced by the osteocytes per unit bone volume can be defined as: 
$S_{\mathrm{oc}}(\boldsymbol{x})=\int_{0}^{2 \pi} d \varphi \int_{0}^{\pi / 2} \frac{2 r_{\mathrm{p}}}{r_{\mathrm{c}}^{2}} \rho_{\mathrm{c}}(\boldsymbol{n}) \mid \overline{\tau_{\mathrm{p}}(\boldsymbol{n}) \mid} \sin \theta d \theta$

where, $\theta$ is the angle between the $x_{3}$-axis in the arbitrarily given Cartesian coordinate system $\left(x_{1}, x_{2}, x_{3}\right)$ and the vector $\boldsymbol{n}, \varphi$ is the angle between the $x_{1}$-axis and the projection of $\boldsymbol{n}$ onto the $x_{1} x_{2}$-plane measured counterclockwise, and $\overline{\left|\tau_{\mathrm{p}}(\boldsymbol{n})\right|}$ is the time-averaged shear stress in a day. Though the volume fraction of canaliculi $\rho_{\mathrm{c}}(\boldsymbol{n})$ depends on their orientation, in this modeling, the cell processes were assumed to extend isotropically, i.e., $\rho_{\mathrm{c}}(\boldsymbol{n})=\rho_{\mathrm{c}}=1 / 2 \pi$, for simplicity.

\section{Intercellular communication}

The biochemical signals produced by osteocytes are delivered to the osteoblasts and osteoclasts on the trabecular surface. The intercellular communication of biochemical signals through the network system is illustrated in Fig. 3. Assuming that the cell on a particular trabecular surface location receives the signals only from the osteocytes within a limited region $\Omega$ and the cellular communication depends on the distance between cells, the total stimulus $S_{\text {sf }}$ at position $\boldsymbol{x}_{\mathrm{sf}}$ on the trabecular surface is defined as $S_{\mathrm{sf}}\left(\boldsymbol{x}_{\mathrm{sf}}\right)=\int_{\Omega} w(l) S_{\mathrm{oc}}(\boldsymbol{x}) d \Omega \quad$ with $w(l)=1-l / l_{\mathrm{L}}\left(l \leq l_{\mathrm{L}}\right)$,

where $w(l)$ is a weight function that describes the decay in signal intensity relative to the distance $l$ and $l_{\mathrm{L}}$ is the maximum distance for intercellular communication. The total stimulus $S_{\text {sf }}$ is a positive scalar function, indicating cellular activity on the trabecular surface. 


\section{Trabecular surface remodeling}

The self-regulation of bone mass is achieved by osteoclastic bone resorption and osteoblastic bone formation. In this study, it was assumed that activities of osteoclasts and osteoblasts are regulated by the decrease and increase in the mechanical stimulus from the physiological equilibrium condition, respectively. Regarding the total stimulus $S_{\mathrm{sf}}\left(\boldsymbol{x}_{\mathrm{sf}}\right)$ as the driving force for bone remodeling, the relationship between the rate of trabecular surface remodeling $\dot{M}$ and $S_{\text {sf }}\left(\boldsymbol{x}_{\mathrm{sf}}\right)$ can be qualitatively described by the function:

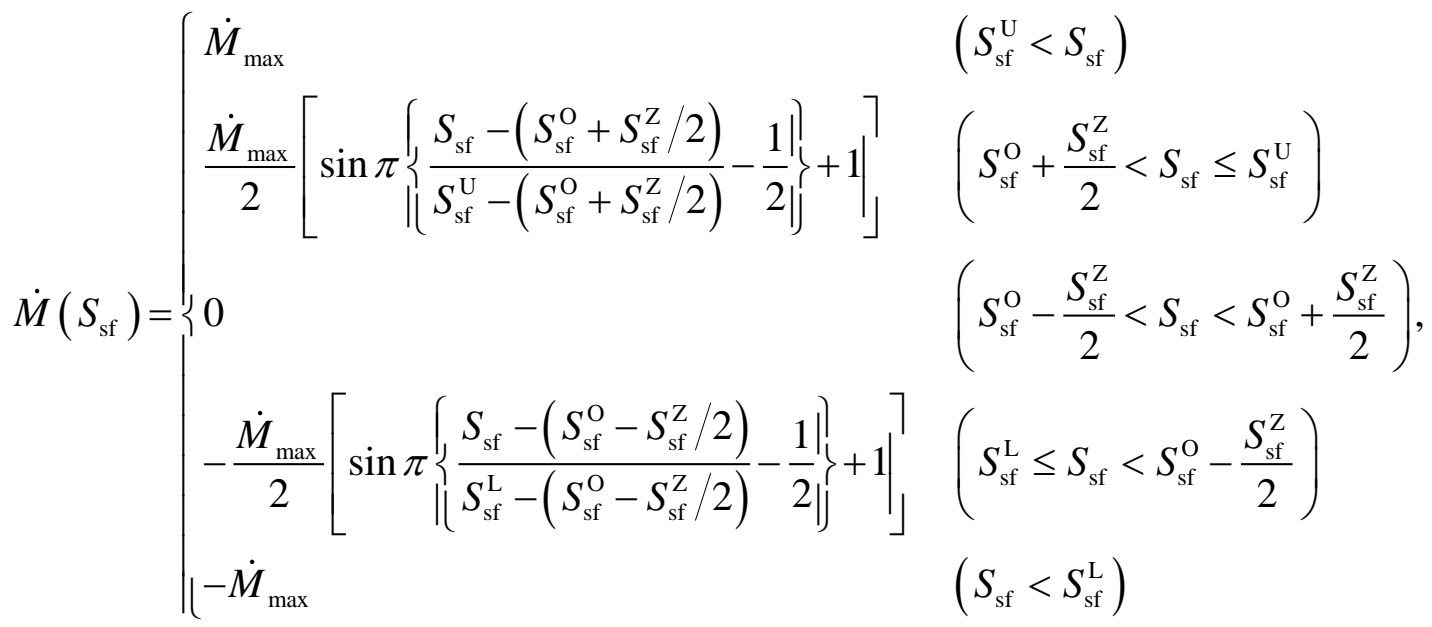

where the mechanical stimulus at the remodeling equilibrium $S_{\mathrm{sf}}=S_{\mathrm{sf}}^{\mathrm{O}}$, the lazy zone $S_{\mathrm{sf}}^{\mathrm{Z}}$, and the maximum formation/resorption rate $\dot{M}_{\max }$ and $-\dot{M}_{\max }$ are considered in the rate equation as shown in Fig. 4. To express trabecular surface movement in the simulation, the level set method (Osher and Sethian, 1988), which is a numerical technique for tracking interfaces and shapes of materials, was employed.

\subsection{Procedures of trabecular bone remodeling simulation}

By applying the mathematical model for trabecular bone remodeling to a voxel finite element model of a trabecula, trabecular surface remodeling was simulated by the following 
procedures.

(1) The initial shape of a trabecula is discretized using cubic voxel finite elements.

(2) The fluid pressure $p$ and its gradient $4 p$ inside the trabecula are calculated based on poroelastic finite element analysis.

(3) The biochemical signal $S_{\text {oc }}$ is calculated by Eq. (3) using the value of fluid-induced shear stress $\tau_{\mathrm{p}}$ derived by Eq. (1).

(4) The total stimulus on the trabecular surface $S_{\mathrm{sf}}$ is calculated by Eq. (4).

(5) The rate of trabecular surface remodeling $\dot{M}$ is determined by Eq. (5).

(6) Based on the value of $\dot{M}$, the shape of the trabecular surface is updated using the level set method, and then the voxel finite element model of the trabecula is recreated.

(7) Unless a remodeling equilibrium is attained, return to procedure (2), where the remodeling equilibrium is accomplished when the changes in the bone volume are sufficiently small.

\subsection{Voxel modeling of a single trabecula}

As a simple trabecular model, a three-dimensional single trabecular model under cyclic uniaxial loading was constructed as shown in Fig. 5. The region for analysis was $a_{1} \times a_{2} \times a_{3}=$ $0.8 \mathrm{~mm} \times 1.6 \mathrm{~mm} \times 1.2 \mathrm{~mm}$, discretized by cubic voxel finite elements with an edge size of $40 \mu \mathrm{m}$. A single trabecula, the diameter was $240 \mu \mathrm{m}$, was set at the center of the region with a skew angle of $30^{\circ}$. The trabecula was modeled as a homogeneous and isotropic poroelastic material (Cowin, 1999; Kameo et al., 2008, 2009) with the material properties listed in Table 
1 (Smit et al., 2002; Beno et al., 2006; Adachi et al., 2010). To impose external loadings, two parallel, $40-\mu \mathrm{m}$-thick plates were added to the upper and lower edges. While the plates have the same material properties as the trabecula, they do not change in the shape during remodeling.

A shear-free condition was applied to the lower plane, and a free leakage of interstitial fluid on the trabecular surfaces was assumed. On the upper plane, cyclic loading $F(t)=$ $\sigma_{3}\left(a_{1} a_{2}\right) \sin 2 \pi f t\left(\sigma_{3}=0.07 \mathrm{MPa}\right)$ was applied along the direction of the $x_{3}$-axis, keeping the $x_{3}$-displacement $u_{3}$ uniform. To investigate the effect of frequency of the applied loading on morphological changes in the trabecula, the loading frequency $f$ was determined as $f=1,5$, and $10 \mathrm{~Hz}$.

The settings of the physiological parameters explained in the previous section were specified as follows. The radius of the osteocytic process $r_{\mathrm{p}}$ and the radius of the canaliculus $r_{c}$ were determined based on the average values measured by You et al. (2004): $r_{\mathrm{p}}=52 \mathrm{~nm}, r_{\mathrm{c}}$ $=129.5 \mathrm{~nm}$. The fiber radius $a_{0}$ and the fiber spacing $\Delta$ were taken as $a_{0}=0.6 \mathrm{~nm}$ and $\Delta=7$ nm (Weinbaum et al., 1994). The maximum remodeling rate $\dot{M}_{\max }=40 \mu \mathrm{m} /$ day (Jaworski and Lok, 1972) was based on the resorption rate of osteoclasts. The maximum distance for intercellular communication $l_{\mathrm{L}}$ was set to $l_{\mathrm{L}}=200 \mu \mathrm{m}$, referring to the in vitro experiment involving the observation of the propagation of calcium signaling between bone cells (Huo et al., 2008; Adachi et al., 2009c). The parameters associated with the mechanical signal, $S_{\mathrm{sf}}^{\mathrm{U}}$, $S_{\mathrm{sf}}^{\mathrm{L}}, S_{\mathrm{sf}}^{\mathrm{O}}$, and $S_{\mathrm{sf}}^{\mathrm{Z}}$, were arbitrarily set to $1.5 \mu \mathrm{N}, 0.5 \mu \mathrm{N}, 1.0 \mu \mathrm{N}$, and $0.2 \mu \mathrm{N}$, respectively. 


\section{Results}

The progress of morphological changes in a single trabecula and the distribution of the average fluid-induced shear stress acting on the osteocytic processes in a day $\overline{\left|\tau_{\mathrm{p}}\right|}$ are shown in Fig. $6 \mathrm{a}-\mathrm{c}$ for the loading frequency $f=1,5$, and $10 \mathrm{~Hz}$, respectively.

In the case $f=1 \mathrm{~Hz}$, as shown in Fig. 6a, relatively-large shear stress was observed at the inner surfaces with an acute angle in the initial state. This enlarged the stimuli $S_{\text {sf }}$ that the trabecular surface cells received from neighboring osteocytes, and bone formation was promoted around both ends, while at the middle of the trabecula, the morphology was almost unchanged. At 3 days, bone resorption dominated on the outer surfaces with an obtuse angle due to small fluid-induced shear stress. After bone remodeling for 6 days, the concentration of fluid-induced shear stress observed in the initial state was relieved. As a result of successive remodeling, the single trabecula aligned along the loading direction.

As shown in Fig. $6 \mathrm{~b}$ and $6 \mathrm{c}$, the fluid-induced shear stress close to the trabecular surfaces in the initial state became larger with increase in the loading frequency. The stimuli $S_{\text {sf }}$ on the surfaces exceeded the physiological equilibrium value, and bone formation occurred on the entire surface in both cases when $f=5$ and $f=10 \mathrm{~Hz}$. While this remodeling enlarged the diameter of the trabecula, the original orientation was preserved. The process of bone remodeling after 3 days was similar to that in the case $f=1 \mathrm{~Hz}$, and as a result, the longitudinal direction of the trabecula coincided with the loading direction. As the imposed loading frequency increased, the diameter of the trabecula in the equilibrium state also increased. 
To evaluate morphological changes in the trabecula quantitatively, the corresponding changes in bone volume fraction and the apparent stiffness during remodeling are plotted in Fig. $7 \mathrm{a}$ and $7 \mathrm{~b}$, where the bone volume fraction refers to the ratio of the volume of the trabecula to that of the total analysis region, and apparent stiffness is defined as the ratio of the loading amplitude $\sigma_{3}$ to the apparent strain on the upper edge $u_{3} / a_{3}$. Regardless of the value of the loading frequency, the bone volume fraction increased slightly at an early stage, and then gradually decreased and converged at the constant value as shown in Fig. 7a. On the other hand, the apparent stiffness of the trabecula increased monotonically and reached a uniform plateau value for all cases as shown in Fig. 7b. As the applied loading frequency increased, the equilibrium values of the bone volume fraction and the apparent stiffness also increased. The relationship between the loading frequency and the bone volume fraction at 30 days is indicated in Fig. 8. This figure also includes the results when $f=3$ and $f=20 \mathrm{~Hz}$. As shown in this figure, the bone volume fraction is not proportional to the loading frequency and tends to converge at a certain value under high loading frequency.

\section{Discussion}

We constructed a mathematical model for trabecular bone remodeling that considers cellular mechanosensing and intercellular signal transmission. To investigate the effect of the applied loading frequency on bone remodeling, we simulated morphological changes of a single trabecula under cyclic uniaxial loading with various frequencies. The results indicated that the diameter of the trabecula in the remodeling equilibrium state increased with the 
increase in the loading frequency, while all the trabeculae reoriented to the loading direction regardless of the value of frequency.

Several computational models for bone remodeling including cellular mechanosensing have been proposed. They assume that the transduction from the external mechanical load to biochemical signals produced by sensor cells is caused by strain energy density (Mullender et al., 1994; Huiskes et al., 2000; Mullender and Huiskes, 1995; Ruimerman at al., 2005), accumulation of micro-damage (Prendergast and Taylor, 1994; McNamara and Prendergast, 2007; Mulvihill and Prendergast, 2008), or nonuniformity of local stress on trabecular surface (Adachi et al., 1997, 2001; Tsubota and Adachi, 2004, 2005, 2006; Tsubota et al., 2002, 2009). These mathematical models, which are based on the phenomenological hypothesis that cellular activities are affected by the mechanical state at the tissue level, can successfully explain bone mechanical adaptation by remodeling. However, the process of cellular mechanosensing is closely related to the mechanical state at the microscopic level. The novelty of our model is that it is possible to consider the mechanical hierarchy from microscopic cellular activities to the macroscopic changes in trabecular morphology by quantifying the mechanical behavior of interstitial fluid in lacuno-canalicular porosity.

As a result of the remodeling simulation when the loading frequency $f=1 \mathrm{~Hz}$, the trabecula rotated toward the loading direction, preserving the total mass as shown in Fig. 7a and $7 \mathrm{~b}$. In general, stiffness as a structure is proportional to the volume of material; nevertheless, the apparent stiffness of the trabecula monotonically increased due to bone remodeling. These results indicate that the trabecula actively adapted to the applied 
mechanical load by changing its own morphology. In addition, Fig. $7 b$ indicates that the increase in the loading frequency derives the increase in the apparent stiffness. Considering that a trabecula subjected to a cyclic loading has a higher risk of fracturing due to fatigue with the increase in the loading frequency, this behavior also could be understood as a functional adaptation of the trabecula to the mechanical environment. The results qualitatively coincide with the experimental findings that a significant bone ingrowth was observed under high loading rate condition (Goldstein et al., 1991). As shown in Fig. 8, in the high frequency range, the bone volume fraction in the equilibrium state does not vary greatly because the interstitial fluid inside the trabecula does not flow easily, and only the osteocytes in the neighborhood of the surfaces contribute to mechanosensing (Kameo et al., 2008, 2009). From a physiological point of view, these results are reasonable. Until date, we cannot verify the validity of our results quantitatively by a comparison with the experimental observations because of the difficulty in observing the morphological changes in a single trabecula under a controlled mechanical environment in vivo. However, if we could conduct a remodeling simulation based on the same mathematical model for a cancellous bone including multiple trabeculae, it would be possible to compare the simulation results with the results of quantitative experiments with respect to the cancellous bone adaptation (Goldstein et al., 1991; Guldberg et al., 1997a, b).

The setting of the mechanical signal parameters introduced in Eq. (5), i.e., $S_{\mathrm{sf}}^{\mathrm{U}}, S_{\mathrm{sf}}^{\mathrm{L}}$, $S_{\mathrm{sf}}^{\mathrm{O}}$ and $S_{\mathrm{sf}}^{\mathrm{Z}}$, is an important issue in this simulation because it significantly affects the bone remodeling process. The parameter $S_{\mathrm{sf}}^{\mathrm{O}}$ is the point of remodeling equilibrium, regulating the 
balance between bone resorption and formation. With the increase in $S_{\text {sf }}^{\mathrm{O}}$, resorption becomes more dominant than formation. The parameter $S_{\mathrm{sf}}^{\mathrm{Z}}$ is the width of the lazy zone (Carter, 1984; Huiskes et al., 1987), representing a remodeling rate sensitivity to the stimulus near the remodeling equilibrium. As the width of the lazy zone $S_{\mathrm{sf}}^{\mathrm{Z}}$ increases, the changes in bone volume are more suppressed. The morphological changes in a trabecula are more sensitive to the parameter set of $S_{\mathrm{sf}}^{\mathrm{O}}$ and $S_{\mathrm{sf}}^{\mathrm{Z}}$ than that of $S_{\mathrm{sf}}^{\mathrm{U}}$ and $S_{\mathrm{sf}}^{\mathrm{L}}$, which describe the remodeling rate sensitivity to the stimulus around the lazy zone. Though these parameters should have to be determined by comparisons with experimental observations, we set them arbitrarily due to the lack of the quantitative experimental results. The set of mechanical signal parameters used here is a case study aimed at explaining physiological bone remodeling; this simulation will also be able to express pathological bone remodeling, such as osteoporosis and osteopetrosis, by choosing the appropriate values of model parameters.

The limitation of our model is that we assumed that the material properties of trabeculae were isotropic. The bone matrix possesses anisotropic mechanical properties due to its microscopic structures. In addition, the observation of a trabecular cross section indicates that the lacuno-canalicular network system has a strong anisotropy that is perpendicular to the trabecular surfaces (Kameo et al., 2010). To investigate their effects on the process of bone remodeling, it will be necessary to resolve the contribution of this anisotropy to the mathematical model. In spite of the above limitations, our mathematical model for trabecular bone remodeling can predict bone functional adaptation successfully. Bone remodeling is a self-regulatory process realized by metabolic activities of many cells at the osteonal and 
trabecular level, but the mechanism is still unclear. By refining our model with the progress of future experimental and theoretical studies, this computational simulation will be an extremely helpful method to clarify the mechanism of bone adaptation by remodeling.

\section{Acknowledgements}

This study was partially supported by a Grant-in-Aid for Specially Promoted Research (20001007) from the Ministry of Education, Culture, Sports, Science and Technology of Japan, and by a Grant-in-Aid from the Japan Society for the Promotion of Science Fellows (201475). 


\section{References}

Adachi, T., Aonuma, Y., Ito, S., Tanaka, M., Hojo, M., Takano-Yamamoto, T., Kamioka, H., 2009a. Osteocyte calcium signaling response to bone matrix deformation. J. Biomech. 42, 2507-2512.

Adachi, T., Aonuma, Y., Tanaka, M., Hojo, M., Takano-Yamamoto, T., Kamioka, H., 2009b. Calcium response in single osteocytes to locally applied mechanical stimulus: Differences in cell process and cell body. J. Biomech. 42, 1989-1995.

Adachi, T., Aonuma, Y., Taira, K., Hojo, M., Kamioka, H., 2009c. Asymmetric intercellular communication between bone cells: Propagation of the calcium signaling. Biochem. Biophys. Res. Commun. 389, 495-500.

Adachi, T., Kameo, Y., Hojo, M., 2010. Trabecular bone remodeling simulation considering osteocytic response to fluid-induced shear stress. Phil. Trans. R. Soc. A 368, 2669-2682.

Adachi, T., Tomita, Y., Sakaue, H., Tanaka, M., 1997. Simulation of trabecular surface remodeling based on local stress nonuniformity. JSME Int. J. 40C, 782-792.

Adachi, T., Tsubota, K., Tomita, Y., Hollister, S.J., 2001. Trabecular surface remodeling simulation for cancellous bone using microstructural voxel finite element models. J. Biomech. Eng. 123, 403-409.

Beno, T., Yoon, Y.J., Cowin, S.C., Fritton, S.P., 2006. Estimation of bone permeability using accurate microstructural measurements. J. Biomech. 39, 2378-2387.

Bonewald, L.F., Johnson, M.L., 2008. Osteocytes, mechanosensing and Wnt signaling. Bone 42, 606-615.

Burger, E.H., Klein-Nulend, J., 1999. Mechanotransduction in bone - Role of the lacuno-canalicular network. FASEB J. 13, S101-S112.

Carter, D.R., 1984. Mechanical loading histories and cortical bone remodeling. Calcif. Tissue Int. 36, S19-S24.

Coelho, P.G., Fernandes, P.R., Rodrigues, H.C., Cardoso, J.B., Guedes, J.M., 2009. Numerical modeling of bone tissue adaptation-A hierarchical approach for bone apparent density and trabecular structure. J. Biomech. 42, 830-837.

Cowin, S.C., 1993. Bone stress adaptation models. J. Biomech. Eng. 115, 528-533.

Cowin, S.C., 1999. Bone poroelasticity. J. Biomech. 32, 217-238.

Cowin, S.C., 2007. The significance of bone microstructure in mechanotransduction. J. Biomech. 40, S105-S109.

Cowin, S.C., Moss-Salentijn, L., Moss, M.L., 1991. Candidates for the mechanosensory system in bone. J. Biomech. Eng. 113, 191-197.

Detournay, E., Cheng, A.H.-D., 1993. Fundamentals of poroelasticity. Comprehensive rock engineering: principles, practice and projects, In: Fairhurst, C. (Ed.), Analysis and Design Method, vol. II, Pergamon Press, Oxford, pp. 113-171. (Chapter 5).

Donahue, H.J., McLeod, K.J., Rubin, C.T., Andersen, J., Grine, E.A., Hertzberg, E.L., Brink, P.R., 1995. Cell-to-cell communication in osteoblastic networks: Cell line-dependent hormonal regulation of gap junction function. J. Bone Miner. Res. 10, 881-889. 
Dunlop, J.W.C., Hartmann, M.A., Brechet, Y.J., Fratzl, P., Weinkamer, R., 2009. New suggestions for the mechanical control of bone remodeling. Calcif. Tissue Int. 85, 45-54.

Fritton, S.P., McLeod, K.J., Rubin, C.T., 2000. Quantifying the strain history of bone: spatial uniformity and self-similarity of low-magnitude strains. J. Biomech. 33, 317-325.

Fritton, S.P., Weinbaum, S., 2009. Fluid and solute transport in bone: flow-induced mechanotransduction. Annu. Rev. Fluid Mech. 41, 347-374.

Gerhard, F.A., Webster, D.J., van Lenthe, G.H., Muller, R., 2009. In silico biology of bone modelling and remodelling: adaptation. Phil. Trans. R. Soc. A 367, 2011-2030.

Goldstein, S.A., Matthews, L.S., Kuhn, J.L., Hollister, S.J., 1991. Trabecular bone remodeling: An experimental model. J. Biomech. 24, 135-150.

Guldberg, R.E., Caldwell, N.J., Guo, W.E., Goulet, R.W., Hollister, S.J., Goldstein, S.A., 1997a. Mechanical stimulation of tissue repair in the hydraulic bone chamber. J. Bone Miner. Res. 12, 1295-1302.

Guldberg, R.E., Richards, M., Caldwell, N.J., Kuelske, C.L., Goldstein, S.A., 1997b. Trabecular bone adaptation to variations in porous-coated implant topology. J. Biomech. 30, 147-153.

Han, Y., Cowin, S.C., Schaffler, M.B., Weinbaum, S., 2004. Mechanotransduction and strain amplification in osteocyte cell processes and flow across the endothelial glycocalyx. Proc. Natl. Acad. Sci. U.S.A. 101, 16689-16694.

Huiskes, R., Weinans, H., Grootenboer, H.J., Dalstra, M., Fudala, B., Slooff, T.J., 1987. Adaptive bone-remodeling theory applied to prosthetic-design analysis. J. Biomech. 20, 1135-1150.

Huiskes, R., Ruimerman, R., Van Lenthe, G.H., Janssen, J.D., 2000. Effects of mechanical forces on maintenance and adaptation of form in trabecular bone. Nature 405, 704-706.

Huo, B., Lu, X.L., Hung, C.T., Costa, K.D., Xu, Q., Whitesides, G.M., Guo, X.E., 2008. Fluid flow induced calcium response in bone cell network. Cell. Mol. Bioeng. 1, 58-66.

Jang, I.G., Kim, I.Y., 2008. Computational study of Wolff's law with trabecular architecture in the human proximal femur using topology optimization. J. Biomech. 41, 2353-2361.

Jaworski, Z.F., Lok, E., 1972. The rate of osteoclastic bone erosion in haversian remodeling sites of adult dogs rib. Calcif. Tissue Res. 10, 103-112.

Kameo, Y., Adachi, T., Hojo, M., 2008. Transient response of fluid pressure in a poroelastic material under uniaxial cyclic loading. J. Mech. Phys. Solids 56, 1794-1805.

Kameo, Y., Adachi, T., Hojo, M., 2009. Fluid pressure response in poroelastic materials subjected to cyclic loading. J. Mech. Phys. Solids 57, 1815-1827.

Kameo, Y., Adachi, T., Hojo, M., 2010. Estimation of bone permeability considering the morphology of lacuno-canalicular porosity. J. Mech. Behav. Biomed. Mater. 3, 240-248.

Kamioka, H., Honjo, T., Takano-Yamamoto T., 2001. A three-dimensional distribution of osteocyte processes revealed by the combination of confocal laser scanning microscopy and differential interface contrast microscopy. Bone 28, 145-149. 
Kamioka, H., Murshid, S.A., Ishihara, Y., Kajimura, N., Hasegawa, T., Ando, R., Sugawara, Y., Yamashiro, T., Takaoka, A., Takano-Yamamoto T., 2009. A method for observing silver-stained osteocytes in situ in 3- $\mu \mathrm{m}$ sections using ultra-high voltage electron microscopy tomography. Microsc. Microanal. 15, 377-383.

Klein-Nulend, J., Bacabac, R.G., Mullender, M.G. 2005. Mechanobiology of bone tissue. Pathol. Biol. 53, 576-580.

Klein-Nulend, J., Semeins, C.M., Ajubi, N.E., Nijweide, P.J., Burger, E.H., 1995a. Pulsating fluid flow increases nitric oxide (NO) synthesis by osteocytes but not periosteal fibroblasts - Correlation with prostaglandin upregulation. Biochem. Biophy. Res. Commun. 217, 640-648.

Klein-Nulend, J., van der Plas, A., Semeins, C.M., Ajubi, N.E., Frangos, J.A., Nijweide, P.J., Burger, E.H., 1995b. Sensitivity of osteocytes to biomechanical stress in vitro. FASEB J. 9, 441-445.

Knothe Tate, M.L., Adamson, J.R., Tami, A.E., Bauer, T.W., 2004. The osteocyte. Int. J. Biochem. Cell Biol. 36, 1-8.

Knothe Tate, M.L., Knothe, U., Niederer, P., 1998. Experimental elucidation of mechanical load-induced fluid flow and its potential role in bone metabolism and functional adaptation. Am. J. Med. Sci. 316, 189-195.

Lanyon, L.E., 1984. Functional strain as a determinant for bone remodeling. Calcif. Tissue Int. 36 (Suppl. 1), S56-S61.

Manfredini, P., Cocchetti, G., Maier, G., Redaelli, A., Montevecchi, F.M., 1999. Poroelastic finite element analysis of a bone specimen under cyclic loading. J. Biomech. 32, $135-144$.

Martinez-Reina, J., Garcia-Aznar, J.M., Dominguez, J., Doblare, M., 2009. A bone remodelling model including the directional activity of BMUs. Biomech. Model Mechanobiol. 8, 111-127.

McNamara, L.M., Prendergast, P.J., 2007. Bone remodelling algorithms incorporating both strain and microdamage stimuli. J. Biomech. 40, 1381-1391.

Mullender, M.G., Huiskes, R., 1995. Proposal for the regulatory mechanism of Wolff's law. J. Orthop. Res. 13, 503-512.

Mullender, M.G., Huiskes, R., 1997. Osteocytes and bone lining cells: Which are the best candidates for mechano-sensors in cancellous bone? Bone 20, 527-532.

Mullender, M.G., Huiskes, R., Weinans, H., 1994. A physiological approach to the simulation of bone remodeling as a self-organizational control process. J. Biomech. 27, 1389-1394.

Mulvihill, B.M., Prendergast, P.J., 2008. An algorithm for bone mechanoresponsiveness: implementation to study the effect of patient-specific cell mechanosensitivity on trabecular bone loss. Comput. Methods Biomech. Biomed. Engin. 11, 443-451.

Osher, S., Sethian, J.A., 1988. Fronts propagating with curvature-dependent speed: algorithms based on Hamilton-Jacobi formulation. J. Comput. Phys. 79, 12-49. 
Parfitt, A.M., 1994. Osteonal and hemi-osteonal remodeling: The spatial and temporal framework for signal traffic in adult human bone. J. Cell. Biochem. 55, 273-286.

Prendergast, P.J., Taylor, D., 1994. Prediction of bone adaptation using damage accumulation. J. Biomech. 27, 1067-1076.

Ruimerman, R., Hilbers, P., van Rietbergen, B., Huiskes, R., 2005. A theoretical framework for strain-related trabecular bone maintenance and adaptation. J. Biomech. 38, 931-941.

Smit, T.H., Huyghe, J.M., Cowin, S.C., 2002. Estimation of the poroelastic parameters of cortical bone. J. Biomech. 35, 829-835.

Sugawara, Y., Kamioka, H., Honjo, T., Tezuka, K., Takano-Yamamoto, T., 2005. Three-dimensional reconstruction of chick calvarial osteocytes and their cell processes using confocal microscopy. Bone 36, 877-883.

Tsay R.Y., Weinbaum, S., 1991. Viscous flow in a channel with periodic cross-bridging fibers: exact solutions and Brinkman approximation. J. Fluid Mech. 226, 125-148.

Tsubota, K., Adachi, T., 2004. Change in the fabric and compliance tensors of cancellous bone due to trabecular surface remodeling, predicted by a digital image-based model. Comput. Methods Biomech. Biomed. Engin. 7, 187-192.

Tsubota, K., Adachi, T., 2005. Spatial and temporal regulation of cancellous bone structure: Characterization of a rate equation of trabecular surface remodeling. Med. Eng. Phys. 27, 305-311.

Tsubota, K., Adachi, T., 2006. Computer simulation study on local and integral mechanical quantities at single trabecular level as candidates of remodeling stimuli. J. Biomech. Sci. Eng. 1, 124-135.

Tsubota, K., Adachi, T., Tomita, Y., 2002. Functional adaptation of cancellous bone in human proximal femur predicted by trabecular surface remodeling simulation toward uniform stress state. J. Biomech. 35, 1541-1551.

Tsubota, K., Suzuki, Y., Yamada, T., Hojo, M., Makinouchi, A., Adachi, T., 2009. Computer simulation of trablecular remodeling in human proximal femur using large-scale voxel FE models: Approach to understanding Wolff's law. J. Biomech. 42, 1088-1094.

Wang, Y., McNamara, L.M., Schaffler, M.B., Weinbaum, S., 2007. A model for the role of integrins in flow induced mechanotransduction in osteocytes. Proc. Natl. Acad. Sci. U.S.A. 104, 15941-15946.

Weinbaum, S., Cowin, S.C., Zeng, Y., 1994. A model for the excitation of osteocytes by mechanical loading-induced bone fluid shear stresses. J. Biomech. 27, 339-360.

Weinbaum, S., Zhang, X., Han, Y., Vink, H., Cowin, S.C., 2003. Mechanotransduction and flow across the endothelial glycocalyx. Proc. Natl. Acad. Sci. U.S.A. 100, 7988-7995.

You, L., Cowin, S.C., Schaffler, M.B., Weinbaum, S., 2001. A model for strain amplification in the actin cytoskeleton of osteocytes due to fluid drag on pericellular matrix. J. Biomech. 34, 1375-1386.

You, L.D., Weinbaum, S., Cowin S.C., Schaffler M.B., 2004. Ultrastructure of the osteocyte process and its pericellular matrix. Anat. Rec. A 278A, 505-513. 
(a)

(b)

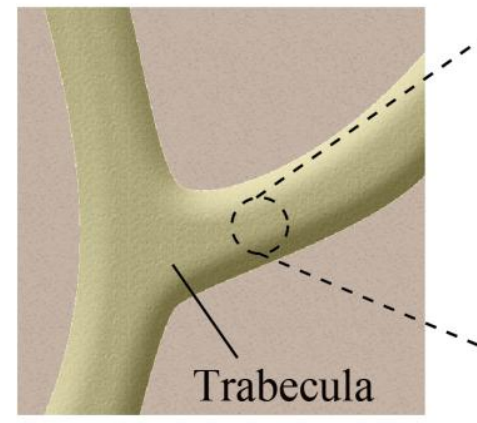

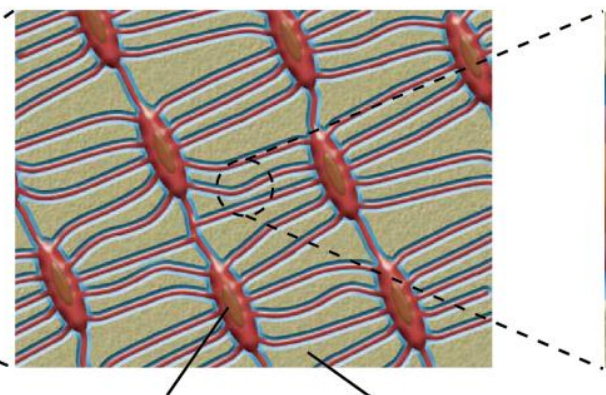

Osteocyte Bone matrix (c)

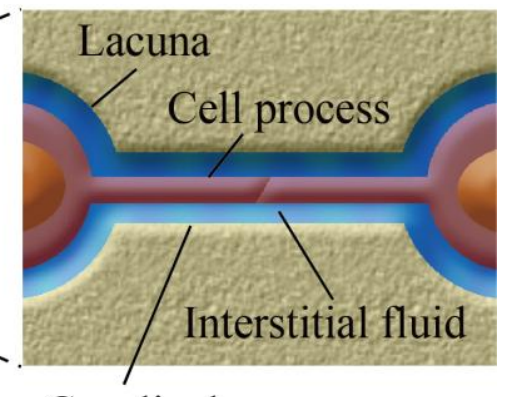

Canaliculus

\section{Cellular}

Mechanical loading mechanosenseing $p, \nabla p$

Change of structure

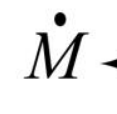

Trabecular surface remodeling<smiles>[As]C1C[As]1</smiles>

\section{communication}

Intercellular

Fig. 1. Modeling framework of trabecular remodeling. This framework considers the mechanical hierarchy from the microscopic to macroscopic level including the (a) trabecular, (b) osteocyte network, and (c) lacuno-canalicular levels.
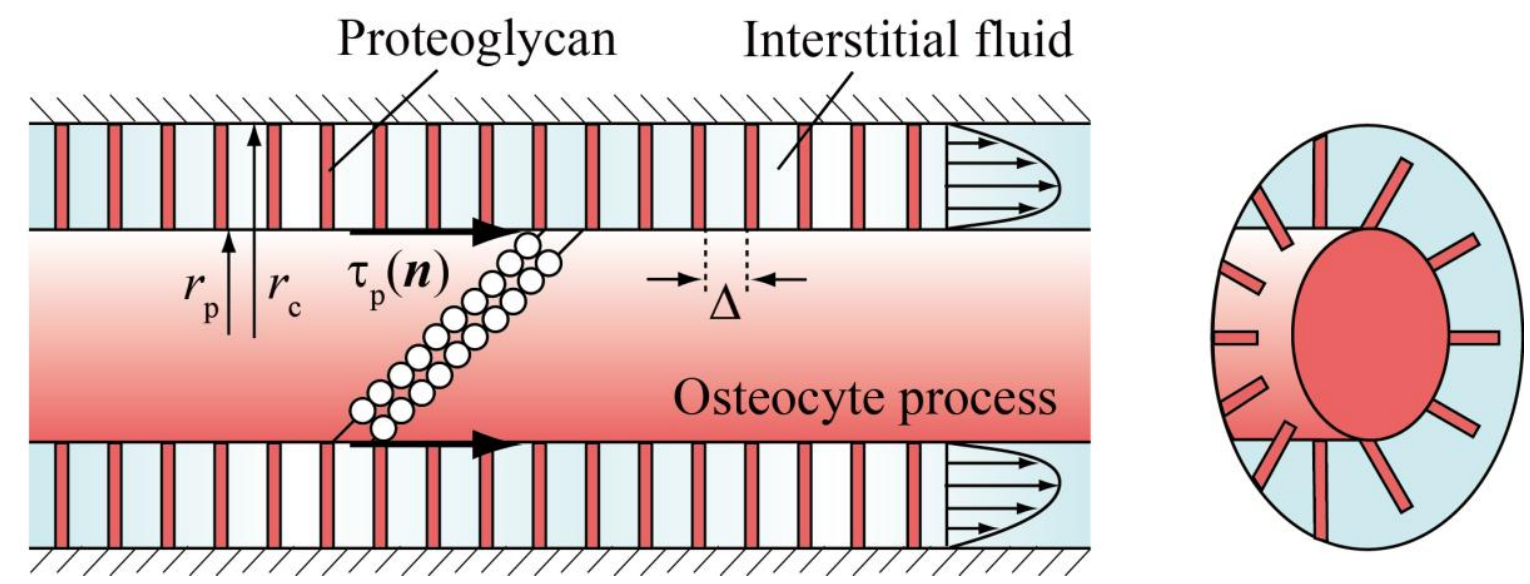

Fig. 2. Modeling of the interstitial fluid flow in an annular canaliculus. (This figure is modified from Weinbaum et al., (1994) with the kind permission of Prof. Sheldon Weinbaum and Elsevier.) 


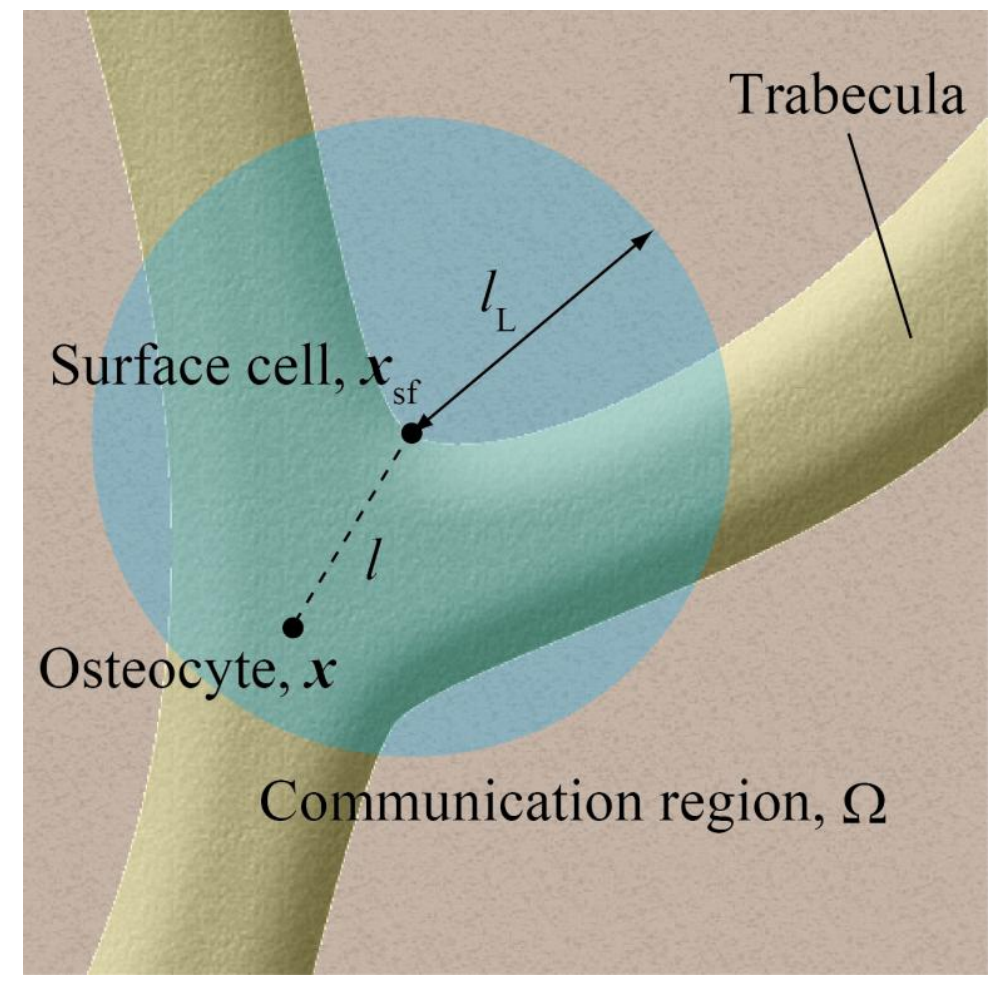

Fig. 3. Modeling of intercellular communication.

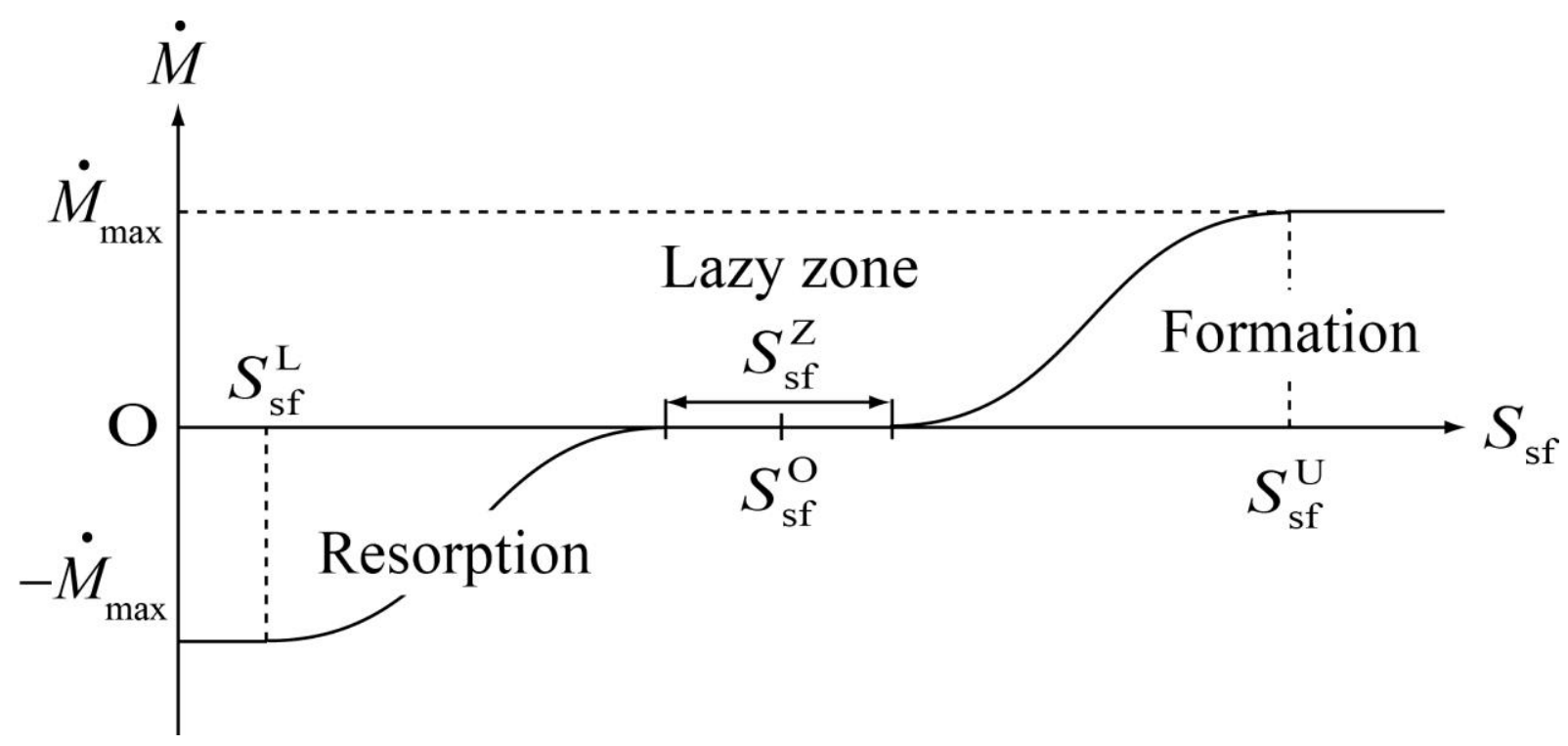

Fig. 4. Relationship between the stimulus $S_{\text {sf }}$ that the cell on the trabecular surface can receive and the trabecular surface remodeling rate $\dot{M}$. 


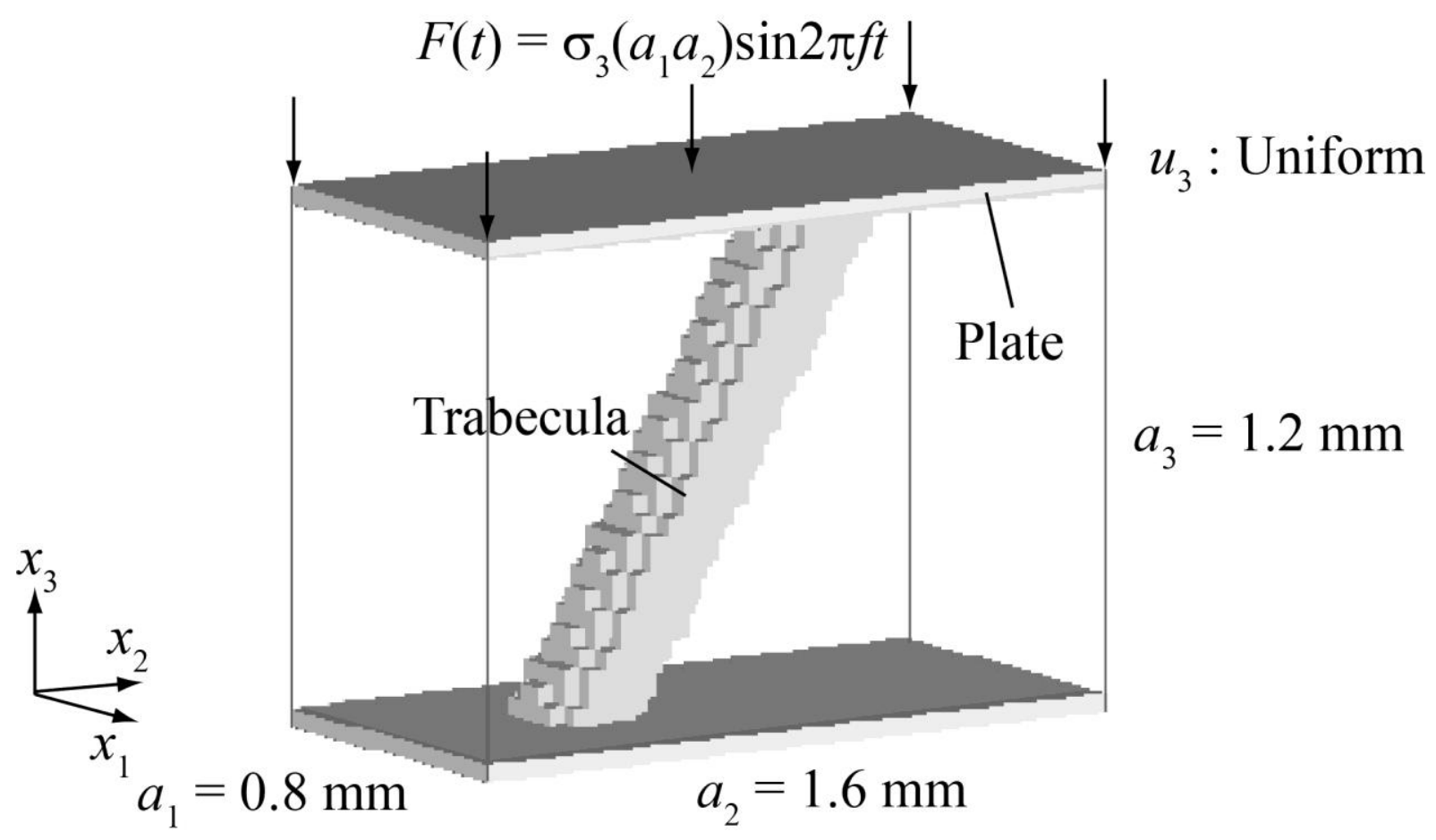

Fig. 5. A model of a single trabecula with a diameter of $240 \mu \mathrm{m}$. On the upper plane, cyclic uniaxial loading is applied along the direction of the $x_{3}$-axis, keeping the $x_{3}$-displacement $u_{3}$ uniform. 
(a)
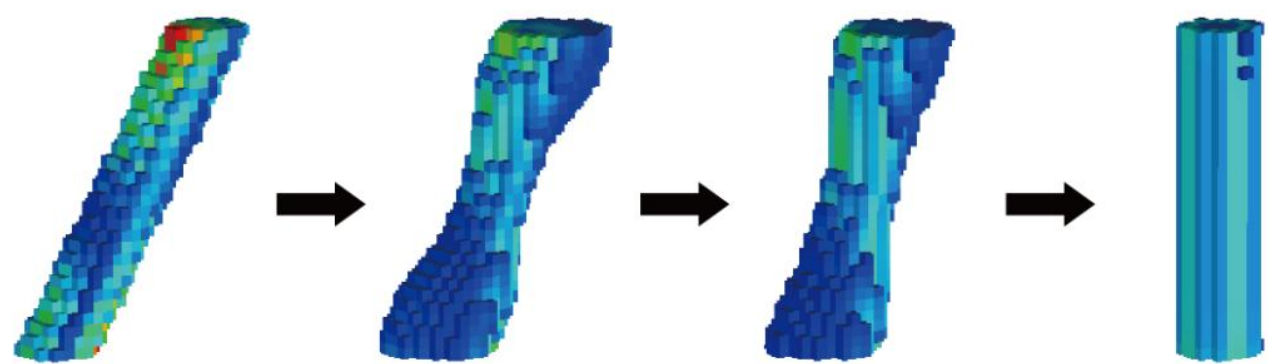

(b)
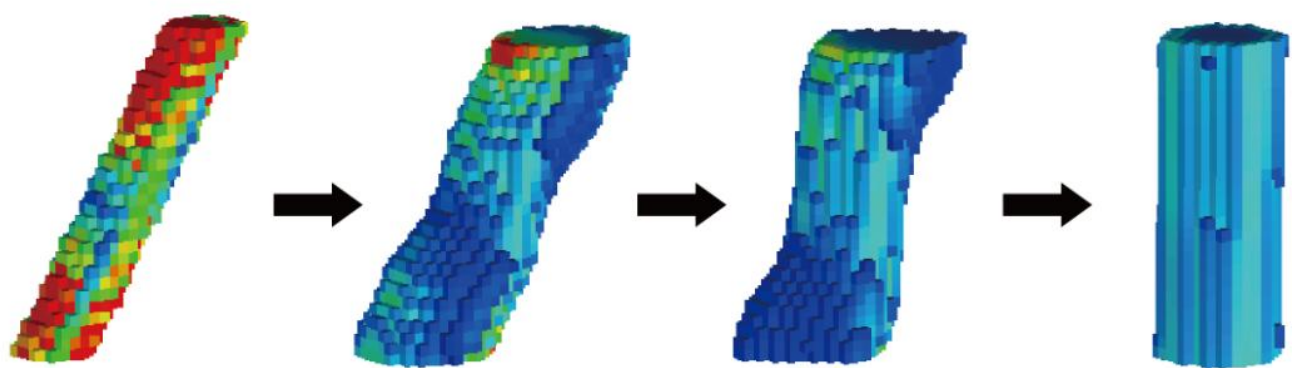

(c)
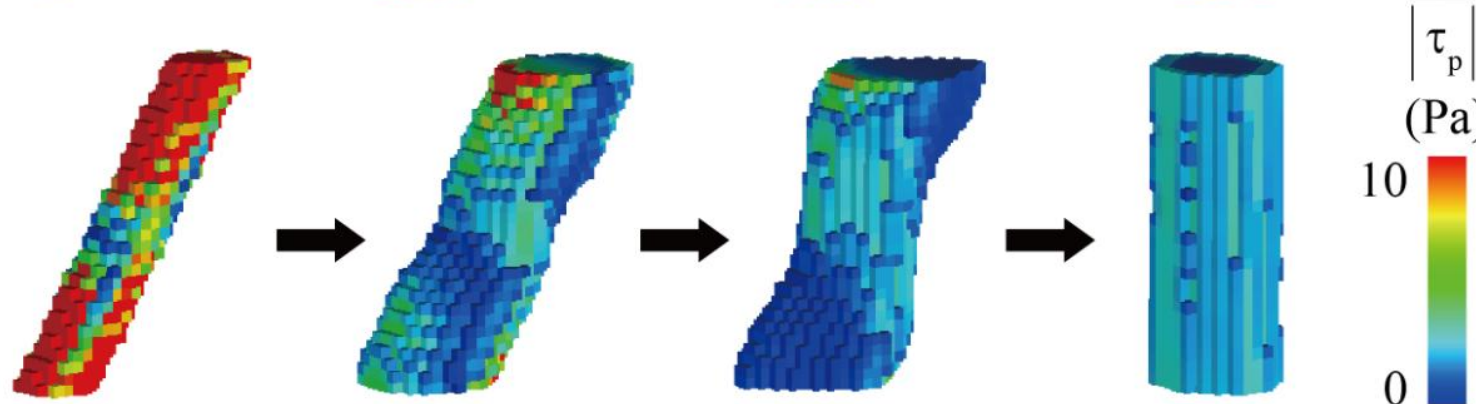

Initial state

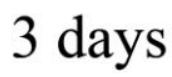

6 days

30 days

Fig. 6. Change in trabecular morphology and average fluid-induced shear stresses $\overline{\left|\tau_{\mathrm{p}}\right|}$ for the applied loading frequencies (a) $f=1 \mathrm{~Hz}$, (b) $f=5 \mathrm{~Hz}$, and (c) $f=10 \mathrm{~Hz}$. 
(a)

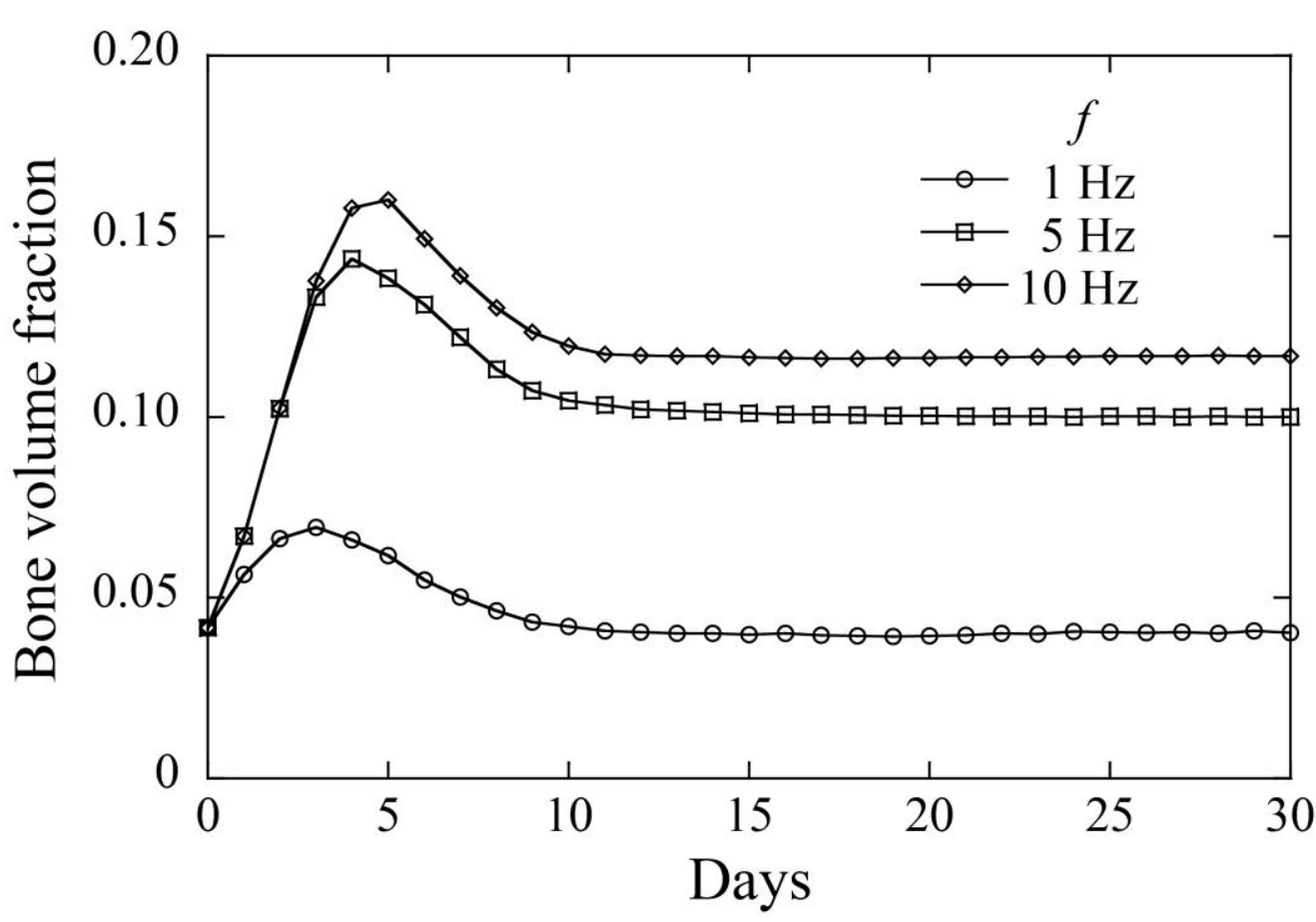

(b)

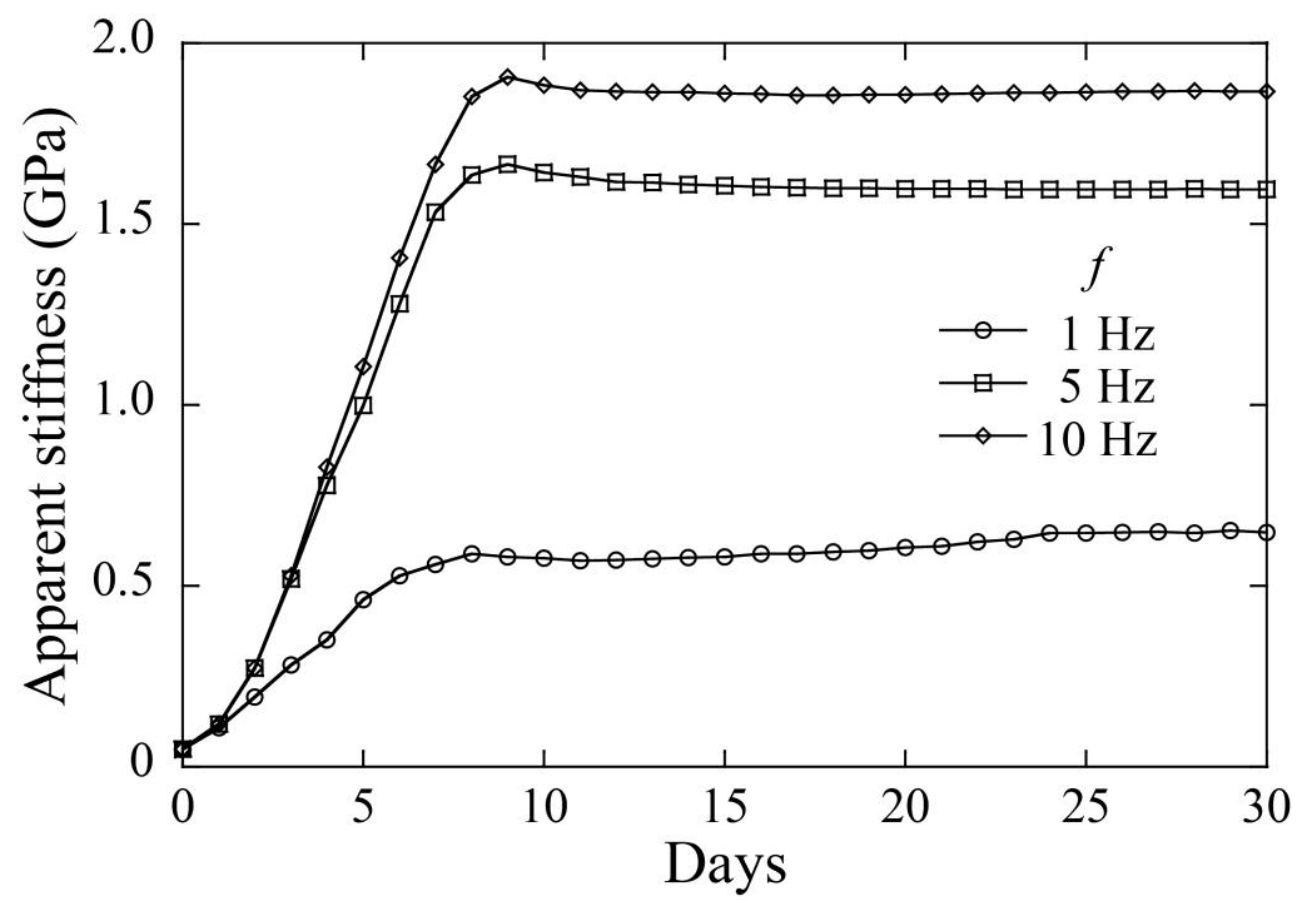

Fig. 7. Quantification of morphological changes in trabecula. (a) Change in bone volume fraction under various loading frequencies, (b) Change in apparent stiffness under various loading frequencies. 


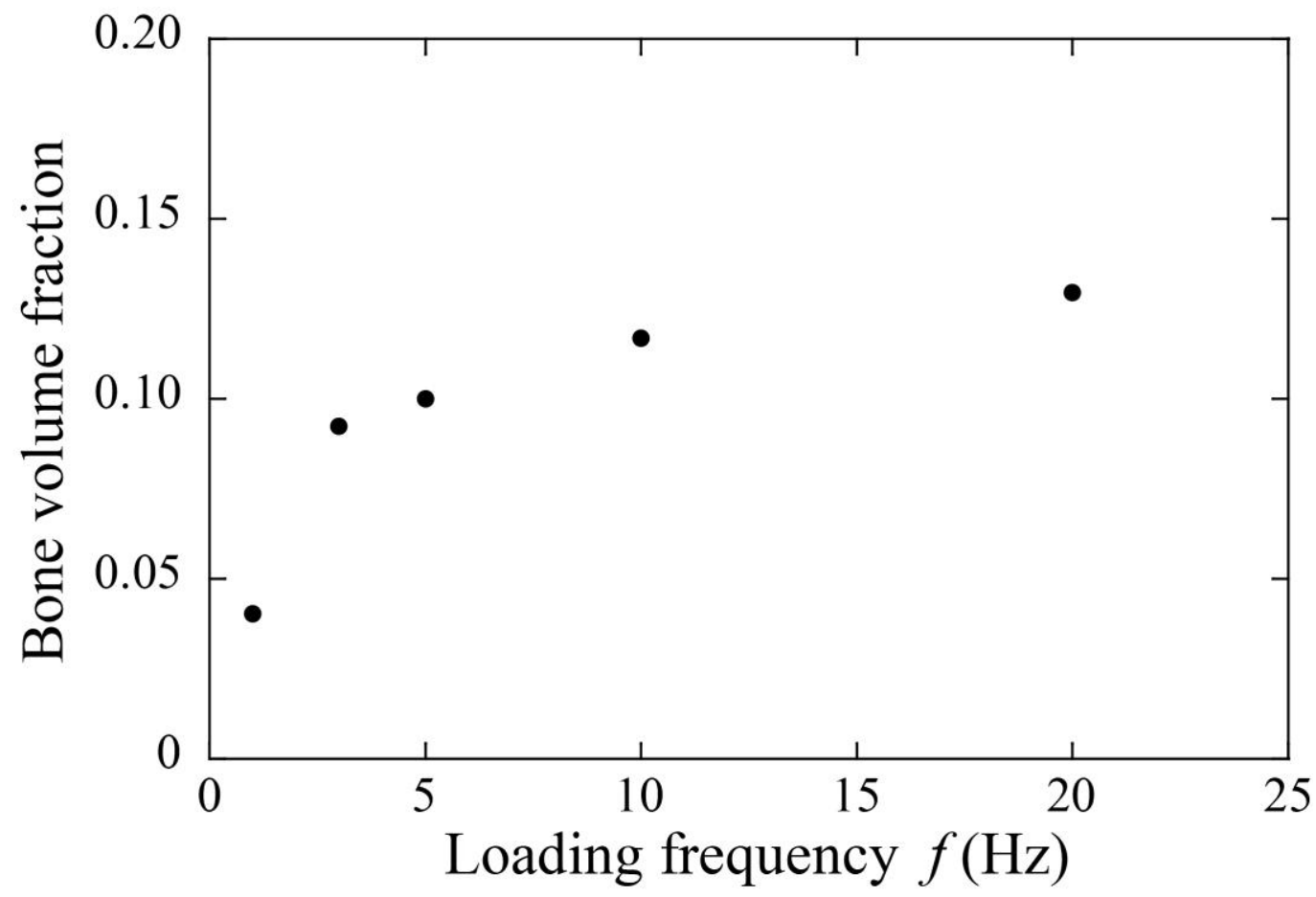

Fig. 8. Relationship between the applied loading frequency $f$ and bone volume fraction.

Table 1. Material properties of trabecula.

\begin{tabular}{ll}
\hline Properties (units) & Value \\
\hline$k$, Permeability $\left(\mathrm{m}^{2}\right)$ & $1.1 \times 10^{-21}$ \\
$\mu$, Viscosity $(\mathrm{Pa} \cdot \mathrm{s})$ & $1.0 \times 10^{-3}$ \\
$G$, Shear modulus $(\mathrm{GPa})$ & 5.94 \\
$\nu$, Drained Poisson's ratio & 0.325 \\
$K_{s}$, Solid bulk modulus $(\mathrm{GPa})$ & 17.66 \\
$K_{f}$, Fluid bulk modulus $(\mathrm{GPa})$ & 2.3 \\
$\phi$, Porosity & 0.05 \\
\hline
\end{tabular}

The value of permeability is estimated by Beno et al. (2006). The other constants are taken from Smit et al. (2002). 